\title{
WILSON DISEASE - CLINICAL FINDINGS \& DIAGNOSIS IN CHILDREN \& ADOLESCENTS
}

\author{
M. Georgieva ${ }^{1}$, D. Konstantinova-Kanazireva ${ }^{1}$, A. Savov ${ }^{4}$, M. Jeleva ${ }^{1}$,

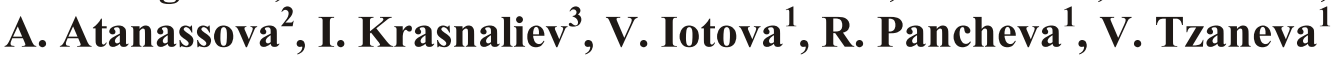 \\ ${ }^{1}$ Dept. of Pediatr., ${ }^{2}$ Clin. of Gastroenterol., ${ }^{3}$ Dept. of General Pathol., University Hospital \\ "St.Marina", Varna, ${ }^{4}$ Genetic Center, Sofia
}

Reviewed by: Assoc. Prof. M. Atanasova

\section{RESUME}

Wilson disease (WD) is an uncommon autosomal recessive condition of impared hepatic copper excretion, resulting in excessive amounts of body copper. Study's aim was to reveal the clinical findings, diagnosis \& treatment of WD in children. In 1987-06 the diagnosis was proved in 21 pts. (3-17y.) (10 M. \& 11 F.) by clinical, laboratory (+specific), neurologic, ultrasonographic, \&/or genetic \& MRI investigation. Eighteen of these children were with hepatic dysfunction: fatigue (17/18), abdominal pain $(16 / 18)$, jaudice $(12 / 18)$, oedema $(6 / 18)$, ascites $(17 / 18)$, hepatomegaly $(18 / 18)$; elevated aminotrasnferases $(18 / 18)$, decreased prothrombin time (16/186) \& serum albumin (14/18) \& cholesterol (5/18). Neurologic manifestations were presented in 3 adolescents: asterixis (3/3), dysarthria (2/3), dysphagia (1/3), rigidity (1/3), choreic movement (1/3) with minimal changes in hepatic function (increased aminotransferases). Twenty of pts. had Kayser-Flaisher corneal ring. Specific examinations showed: decreased ceruloplasmin (19/21), decreased serum copper (16/21), increased urinary excretion of copper for $24 \mathrm{~h}$. (21/21). Test with $1,0 \mathrm{D}$ penicillamine provocated the elevation of urinary excretion of copper from 10-20 times. In 15/18 pts. the mutation in ATP 7B gene is proved. Ultrasound imaged portal hypertensia in 15/21 pts., ascites in 3/8, hepatomegaly in 19/21. MRI showed changes in the basal ganglia in 1 of the pts. with neurologic symptoms. In conclusion the diagnosis of WD is difficult because of the variable clinical manifestation \& laboratory tests. In children \& adolescents with unclear hepatic \&/or neurologic symptoms copper metabolism \& molecular genetic analysis should be examined.

Key words: Wilson disease, children, hepatitis, asterixis, copper metabolism, DNA analysis

\section{BACKGROUND}

Wilson disease (WD) is an uncommon autosomal recessive condition of impaired hepatic copper excretion, resulting in excessive amounts of body copper $(1,2,9)$. It was first described in 1912 by Dr Samuel Alexander Kinnier Wilson as "progressive lenticular degeneration", a familial, lethal neurological disease accompanied by chronic liver disease leading to cirrhosis $(5,8)$. In 1993 the gene of WD was identified -ATP7B, encoding to a metaltransporting P-type ATPase. It is expressed mainly in hepatocytes and functions in the transmembrane transport of copper $(6,7,12)$. WD occurs worldwide - 30 affected individuals per million population $(5,10)$. Clinically WD is manifested as a liver disease or progressive neurological disorder, or psychiatric illness. In children and young adults WD usually presents

Address for correspondence:

M.Georgieva, Dept. of Pediatrics \& Genetics,University Hospital

"St. Marina", Varna 9000, Bulgaria

email: mgeorgieva7@yahoo.com as a liver disease compared to more often neurological engagement in older adults $(1,5)$.

WD was fatal until treatment was developed half a century ago. WD was one of the first liver diseases for which effective pharmacologic treatment was identified. First chelating agent, from 1951, for the treatment of WD was a British anti-lewisite (BAL or imercaptopropanol). Identification and testing of an orally administered chelator, D-penicillamine, by John Walsh in 1956 revolutionized treatment of this disorder. After that zinc salts (1961), trientine (1969), liver transplantation (1982), and tetrathiomolybdate (1984) as treatment options were introduced $(3,4,11,13)$.

Aim of the study was to reveal the clinical findings, diagnosis and treatment of WD in children.

\section{MATERIAL AND METHODS}

In the period 1987-2006 the diagnosis was proved in 21 patients, aged 3 to 17 years. Of them 10 were boys and 11 were girls. The diagnosis was based on clinical and neuro- 
logical complains and signs, laboratory (+specific) testing, ultrasonography and MRI examination, and/or genetic analysis.

\section{RESULTS AND DISCUSSION}

The majority of children with WD presented with predominantly hepatic injury (18 patients) and only a few with neuropsychiatric symptoms (3 patients). Those with leading neuropsychiatric clinical picture presented with either clinically asymptomatic or mild liver involvement. Patients with hepatic form of WD manifested progressive chronic liver disease in the form of either chronic active hepatitis (10 patients) or cirrhosis of the macronodular type (6 patients); 3 patients developed fulminant hepatic failure. The degree of liver involvement was variable, ranging from asymptomatic hepatosplenomegaly with mild elevations of certain liver enzymes ( 3 patients), to complete liver failure (4 patients). Associated symptoms included nonspecific general symptoms - fatigue, abdominal pain; hepatomegaly, ascites and jaundice, and symptoms such as hematemesis and melena, that are caused by portal hypertension ( 2 patients). In general, the younger the age of the patient at symptom onset, the greater the degree of liver involvement $(1,5)$. The children, who presented with fulminant liver failure, were the youngest patients ( 2.5 to 8 years of age). All of them died with the signs of hepato-renal syndrome (Fig. 1).

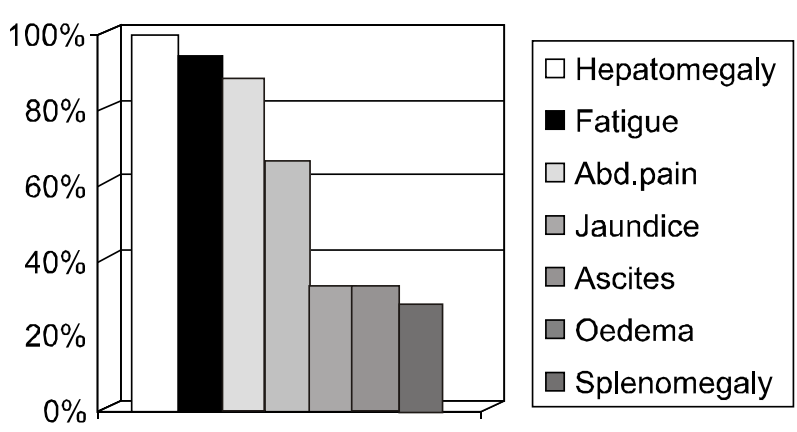

Fig. 1. Clinical findings in WD children with hepatic dysfunction

The 3 patients with neurological form of WD presented with asterixis, dysphagia, rigidity, dysartria and choreic movements (Fig. 2). The mean age of onset of 'neurological WD' is the second to third decade, although it has been reported as late as 72 years of age. Patients commonly present with extrapyramidal, cerebellar and cerebral-related symptoms, in either a subacute or a chronic fashion $(8,12,13)$. The most common initial presentation was bulbar symptoms, characterized by difficulties in talking and swallowing, and drooling. These symptoms were related to dystonia of the bulbar muscles, or pseudobulbar palsy. Abnormal posturing caused by limb dystonia interfered with writing and walking, and features of parkinsonism occured in combination. One child presented with choreic movements. Rarely, patients exhibit chorea of generalized or localized distribution over one half of the body $(4,5,10)$. Myoclonic or tonic-clonic seizure disorders - of either the generalized

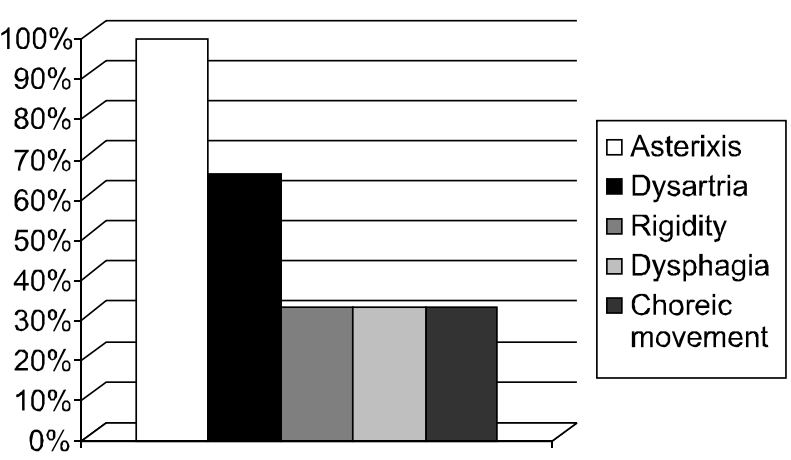

Fig. 2. Clinical findings in children with neurological manifestations of WD

or the partial variety - are seen infrequently $(1,5,7,12)$. The Kayser-Fleischer (KF) ring around the periphery of the cornea is caused by deposition of copper in Descemet's membrane $(5,10)$. This is a characteristic finding observed in most cases of neurological Wilson disease and approximately $50 \%$ of cases with the hepatic form. The ophthalmologic examination of our patients showed presence of KF ring in 9 children with hepatic form and in all neurological cases (Fig. 3).

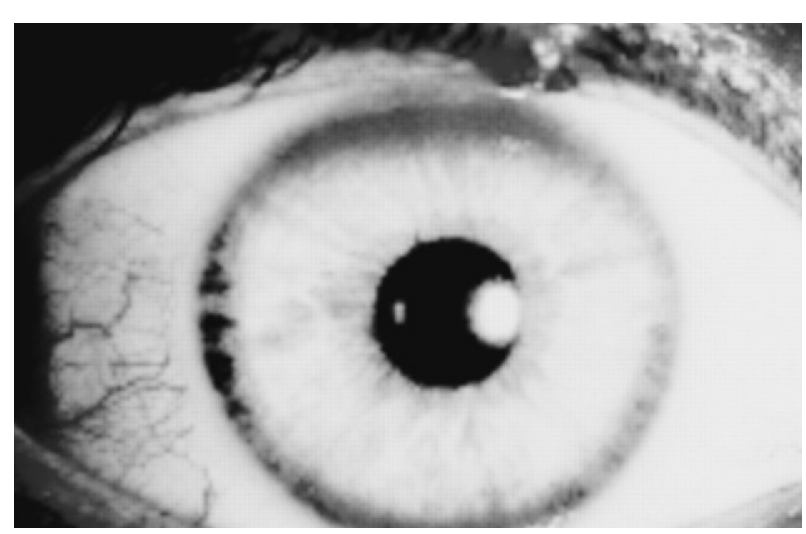

Fig. 3. Keiser Fleisher ring

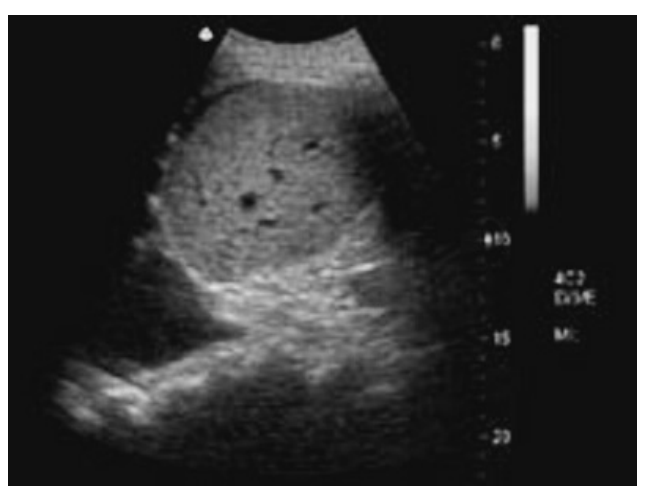

Fig. 4. Ultrasonographic study

The laboratory testing in most of the patients was consistent with abnormal liver function: elevated aminotransfearses, low cholesterol, low albumin, and low protrombine time. 
Ceruloplasmin and total serum copper levels were also decreased while copper excretion in 24-hour urine was increased (Table 1).

Table 1. Laboratory data in children with WD

\begin{tabular}{||l|c|}
\hline \hline Findings & Percentage \\
\hline $\begin{array}{l}\uparrow 24 \mathrm{~h} \text { urinary excretion of } \\
\text { copper }\end{array}$ & $100 \%$ \\
\hline$\downarrow_{\text {serum copper }}$ & $76.19 \%$ \\
\hline$\downarrow$ ceruloplasmin & $90.48 \%$ \\
\hline$\uparrow$ aminotrasnferases & $100 \%$ \\
\hline$\downarrow$ prothrombin time & $88.88 \%$ \\
\hline$\downarrow_{\text {serum albumin }}$ & $77.77 \%$ \\
\hline$\downarrow$ cholesterol & $27.77 \%$ \\
\hline \hline
\end{tabular}

Measurement of the serum ceruloplasmin level is important. Normal values can show some variation depending on the method of estimation (11). Because ceruloplasmin acts as an acute phase reactant as well as a copper transporter, falsely high levels will be seen when patients have active inflammation, are

Table 2. Bacterial and viral testing

\begin{tabular}{|c|c|}
\hline Agent tested & Results \\
\hline Tuberculosis & Negative \\
\hline Toxoplasmosis & Negative \\
\hline Brucellosis & Negative \\
\hline Listeriosis & Negative \\
\hline Syphilis & Negative \\
\hline Kala azar (Leishmania don.) & Negative \\
\hline HBsAg, $\mathrm{HBeAg}$, antiHBe & Negative \\
\hline
\end{tabular}

pregnant, or are taking estrogens. Low levels of ceruloplasmin can also be found in cases of Menkes disease, hereditary aceruloplasminemia, protein-losing enteropathy including celiac disease and severe hepatic insufficiency, and in heterozygous carriers of WD gene (5).

An estimation of 24-hour urinary copper excretion is another reliable test for confirmation of WD (12). In cases of WD, excretion is increased. In children with WD - especially those with the hepatic form - a provocative test for urinary copper excretion using the chelating agent D-penicillamine has been undertaken, with high levels of sensitivity reported.(5). In all our patients, test with $1.0 \mathrm{D}$ penicillamine led to 10-20 times elevation of copper urinary excretion.

All other metabolic, bacterial and viral examinations were negative (Table 2).
Imaging plays an important role in both the diagnosis of WD and the monitoring of patients during treatment.

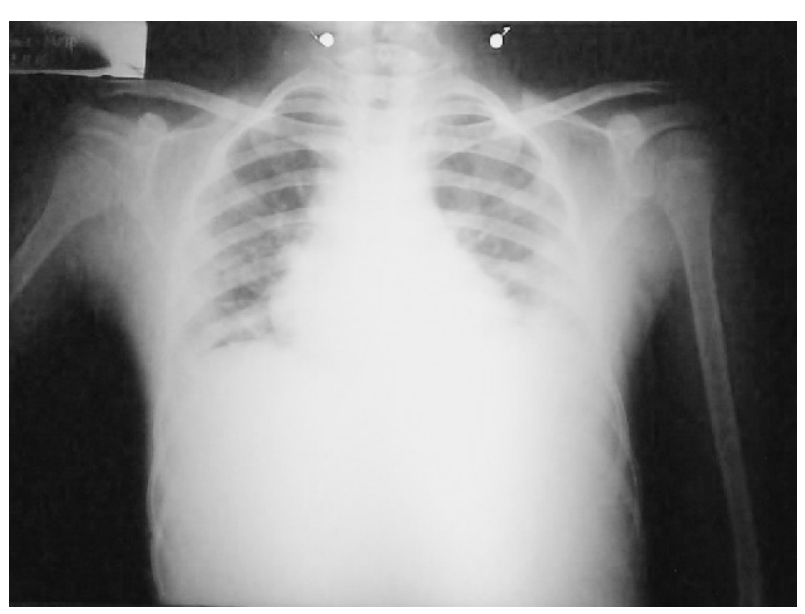

Fig. 5. Chest raentgenogram

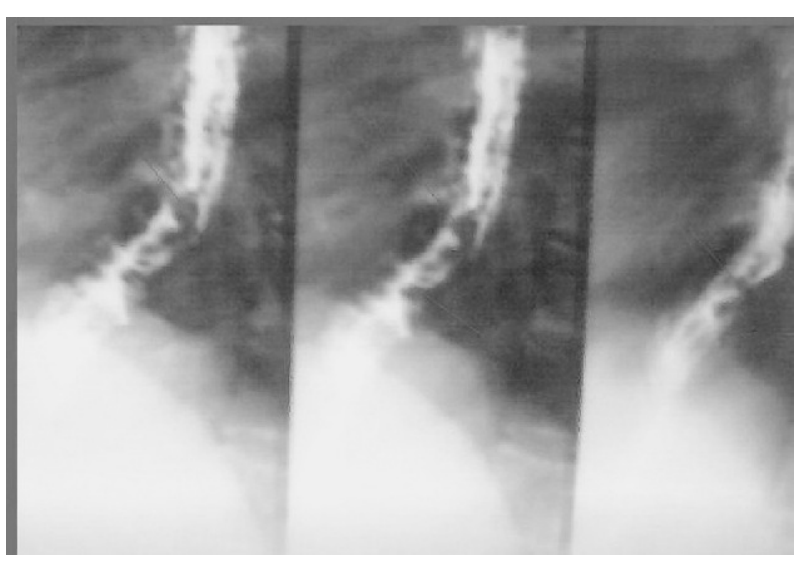

Fig. 6. Ezophageal varicies

Ultrasonography showed enlarged liver with nodular surface, increased echogenicity, thickening of gallbladder wall, enlarged spleen, in some case small volume of ascites (Fig. 4). In 2 children upper GI series showed esophageal varices (Fig. 5); in one child chest X-ray revealed elevated diaphragms and pleural fluid (Fig. 6).

In one of the children MRI showed changes in basal ganglia (Fig. 7). MRI is a very sensitive method for revealing brain abnormalities in $\operatorname{WD}(5,12)$. On T2-weighted images, one-third of cases demonstrate hyperintensity in the basal ganglia, white matter, thalamus or brainstem . These abnormalities are caused by neuronal loss, gliosis, degeneration of fibers, and vacuolization associated with increased water content in the brain (10).

In 3 children we performed liver biopsy - it showed vacuolar degeneration of the hepatocytes, intra- and extra hepatic cholestasis, regenerative nodules of hepatocytes, surrounded by fibrous connective tissue, bridges between portal tracts, lymphocytes, proliferation of bile ducts, large drops steatosis (Fig. 8).

Genetic analysis (DNA analysis) was done. ATP7B gene screening for the most common WD mutations was performed in 18 patients. Fifteen were found homozygotes for 
His1070Gln; the rest were compound-heterozygotes. The use of mutation screening to identify defects in the ATP7B gene can provide unequivocal confirmation of WD in an affected symptomatic or presymptomatic individual

\section{CONCLUSION}

The diagnosis of WD is difficult because of the variable
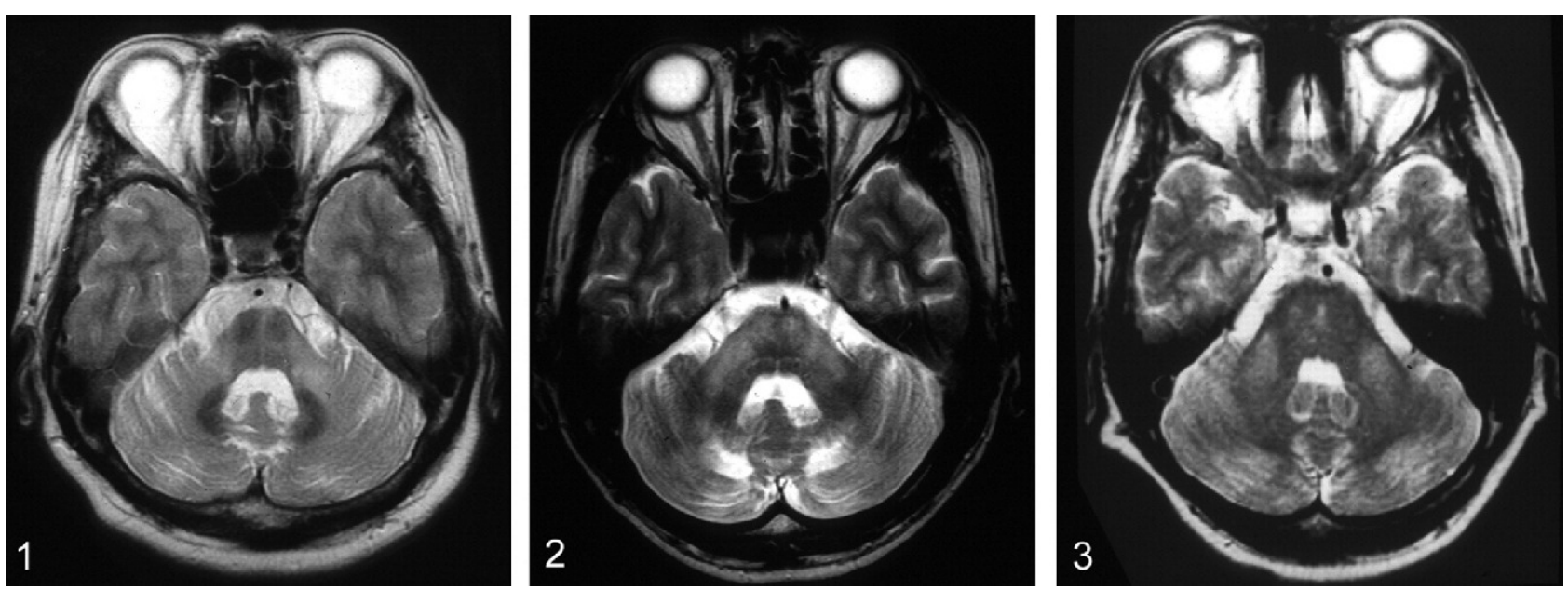

Fig. 7. MRI study

$(6,7,12)$. Monitoring of the presymptomatic individuals and placement on a treatment regimen before the onset of clinical symptoms can then be carried out as appropriate.
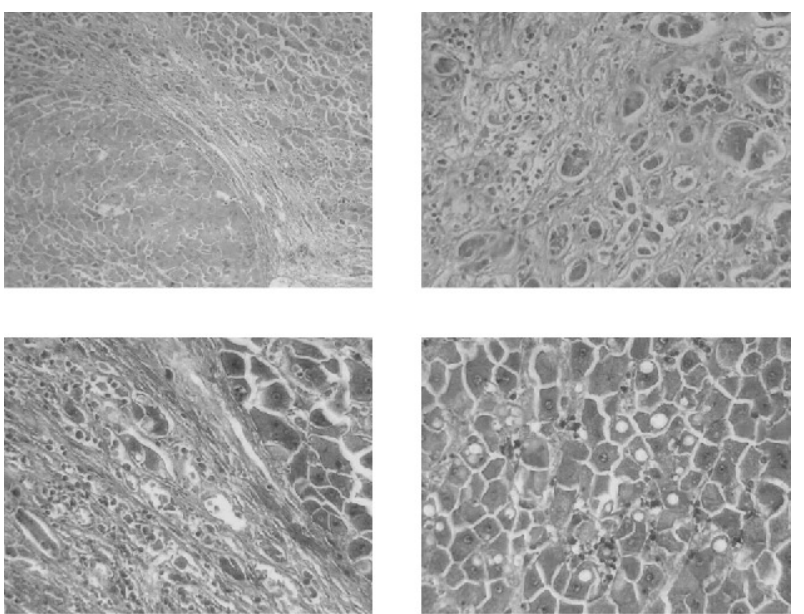

Fig. 8. Liver biopsy in a child with WD

Differential diagnosis of conditions with liver involvement in childhood is very wide. Hepatomegaly and splenomegaly have multiple causes in early childhood most often (but not only) blood and liver diseases. Among liver disorders the following have to be considered:

1. Autoimmune hepatitis

2. Chronic viral hepatitis

3. Bacterial \& spirochetal infections

4. Parasitic diseases

5. $\alpha 1$ antitrypsin deficiency

6. Cystic fibrosis

7. Indian intrahepatic cirrhosis

8. Glycogen storage disease

9. Hereditary hemochromatosis clinical manifestation and laboratory tests. In children and adolescents with unclear hepatic and/or neurological symptoms copper metabolism should be examined and molecular genetic analysis should be performed.

\section{REFERENCES}

1. Коларски. В. Болест на Wilson. В

"Хепатология" Под редакцията на Проф. 3.

Кръстев и Проф. К. Чернев, Изд. Тилия, 1998г., 441-454

2. Brewer G .J. Wilson's disease. Current treatment options in neurology, 2, 2000, 193-204

3. Brewer G .J, F. Askari, M. T. Lorincz et al. Treatment of Wilson disease with ammonium tetrathiomolybdate: IV. Comparison of tetrathiomolybdate $\&$ trientine in a double-blind study of treatment of neurologic presentation of Wilson disease. Arch. Neurol., 63, 2006, 4, 521-5274

4. Brewer G.J. Novel therapeutic approaches to the treatment of Wilson's disease. Expert. Opin. Pharmacother., 7, 2006, 3, 317-324

5. Das S. K. \& K. Ray. Wilson's disease: an update, Nat.Clin.Pract. Neurol., 9, 2006, 2, 482-403

6. De Bie P., P. Muller, C. Wijmenga, L. W. Klomp. Molecular pathogenesis of Wilson \& Menkes disease: correlation of mutations with molecular defects \& disease phenotypes. J. Med. Genet., 44, 2007, 11, 673-688

7. Durand F. Wilson's disease: an old disease keeps its old secrets. Eur. J. Gastroenetrol. Hepatol, 19, 2007, 2, 97-99

8. Ferenci P. Wilson's disease. Clin. Gastroenterol. Hepatol., 3, 2005, 8, 726-733

9. Fink S., M. L. Schilsky. Inherited metabolic disease of the liver. Curr. Opin. Gastrenterol., 23, 2007, 3, 237-243 